\title{
El papel de los hitos de desarrollo pragmático en la detección precoz de trastronos del neurodesarrollo.
}

\section{The role of pragmatics developmental milestones in the early detection of}

\section{neurodevelopmetal disorders.}

\author{
Iria Botana*, Manuel Peralbo**. \\ **Centro Atención Temprana Ordes ,A Coruña , España**Departamento de Psicología, Universidad de la Coruña, España
}

\begin{abstract}
Resumen
El objetivo del presente trabajo es mostrar datos empíricos sobre el valor del componente pragmático como herramienta de detección precoz de las alteraciones del neurodesarrollo. Para ello consideramos los datos extraídos de la aplicación de la escala EDPRA (Botana y Peralbo, 2015), aplicada a un total de 298 niños y niñas de entre 6 y 48 meses. Después del proceso de validación de la escala se detectaron un total de 19 ingresos en Servicios de Atención Temprana, de menores con dificultades asociadas a posibles trastornos del neurodesarrollo. En todos los casos los aspectos pragmáticos del lenguaje, fueron el indicador más temprano y con mayor valor predictivo.
\end{abstract}

Palabras clave: desarrollo pragmático, detección precoz.

\section{Abstract}

The objective of the present work is to show empirical data on the value of the pragmatic component as a tool for the early detection of neurodevelopmental disorders. For this we consider the data extracted from the application of the EDPRA scale (Botana and Peralbo, 2015), to 298 children between 6 and 48 months. Finished the validation process of the scale were detected a total of 19 children with difficulties associated with possible neurodevelopmental disorders in Early Care Services. In all cases, the pragmatic aspects of language were the earliest indicator and with the highest predictive value.

Keywords:pragmatic development, early detección.

\section{Introducción}

Independientemente de su eficacia, la Atención Temprana (en adelante AT) responde a una demanda básica derivada de la identificación de un trastorno o riesgo del mismo. Ante cualquier alteración del estado de salud, por el motivo que sea, se genera una fuerte y comprensible presión para actuar lo más pronto posible (Artigas, 2007) .En el caso de un trastorno del neurodesarrollo, la detección precoz tiene un indudable protagonismo, una detección certera mejora el proceso de AT y con ello el pronóstico del niño. Al hablar de detección precoz históricamente se ha considerado el desarrollo motor como el indicador principal en la detección de un importante número de trastornos de la primera infancia, esto ha sido así durante años, ya que los trastornos que se detectaban, eran aquellos ligados principalmente a la motricidad. Con la propia evolución de la AT el perfil de casos que reciben terapia se ha ido modificando de manera considerable superando en número, los trastornos asociados a la comunicación y el lenguaje al total de los demás trastornos demandantes de AT (Botana y Vilameá, 2014). Esta modificación muestra la necesidad de nuevas herramientas, en las que los hitos de lenguaje y comunicación reciban la observación y análisis que merecen. En esta línea, las nuevas escalas de desarrollo conceden cada vez más espacio al lenguaje, y las escalas propiamente de lenguaje son usadas en más diversidad de alteraciones del desarrollo. Con lo cual, analizar los datos extraídos de la aplicación de escalas propiamente de lenguaje y ver su eficacia como herramientas de detección será un paso más hacia la atención global desde una detección más eficaz y precoz.

En esta línea, el presente trabajo, pretende analizar los datos extraídos durante el proceso de baremación de la escala EDPRA (Botana y Peralbo, 2015), escala de desarrollo pragmático. Durante el mismo se encontraron alertas en los hitos pragmáticos de desarrollo previos a cualquier otro indicador o alerta que posibilitarían la detección temprana de alteraciones en el desarrollo de los menores.

\section{Participantes \\ Método \\ El muestreo se realizó siguiendo un criterio de} conveniencia. Como criterio de exclusión se descartaron aquellos casos en los que pudiera existir sospecha de una alteración que afecte al desarrollo comunicativo, lingüístico o cognitivo de los niños. Además, fueron considerados también criterios de exclusión la existencia de deficiencia auditiva moderada o grave, deficiencia visual severa, síndrome de filiación genética, grandes prematuros, trastorno del neurodesarrollo, trastornos del sistema nervioso central, hospitalización prolongada, institucionalización durante los primeros meses de vida.

La muestra fue extraída de cuatro centros de educación públicos de la provincia de A Coruña (ubicados en la 
comarca de Órdenes) tres centros de primer ciclo de educación infantil y un centro de segundo ciclo de educación infantil, siguiendo los protocolos de actuación pertinentes en materia de investigación en los que se incluyen los consentimientos informados de los padres de los participantes así como de las propias escuelas infantiles.

El tamaño muestral fue $n=298$ y la edad cronológica de los participantes oscilaba entre los 6 meses y los 48 meses. Todos los participantes formaban parte de la muestra utilizada para la validación de la escala EDPRA.

\section{Instrumentos}

\section{Escala de Desarrollo Pragmático (EDPRA).}

La escala EDPRA, contiene tres ejes de evaluación pragmática. Cada eje incluye un máximo de 6 items cuantificables con puntuaciones de $0,1,2$ ó 3 y un procedimiento detallado de aplicación de cada uno (Botana y Peralbo, 2015)

La escala EDPRA dispone de una hoja de respuesta con sus correspondientes ítems para cada rango de edad: 6 a 12 meses, 12 a 24 meses, 24 a 36 meses y 36 a 48 meses. Todas ellas tienen un máximo de 18 ítems descritos a partir de situaciones conocidas para los niños.

\section{Resultados}

Detallamos en la tabla 1 los datos obtenidos durante el proceso de baremación del a escala .

Tabla 1.

Ingresos en SAT posteriores a la aplicación de la escala EDPRA.

\begin{tabular}{ccccc}
\hline Edad & $\mathrm{N}$ & Sexo & $\begin{array}{c}\mathrm{N}^{\circ} \text { casos } \\
\text { con } \\
\text { puntuaciones } \\
\text { bajas }\end{array}$ & $\begin{array}{c}\text { Casos } \\
\text { en SAT }\end{array}$ \\
\hline $6-12 \mathrm{~m}$ & 51 & $\begin{array}{c}26 \mathrm{M} \\
25 \mathrm{~V}\end{array}$ & 4 & 4 \\
$13-24 \mathrm{~m}$ & 99 & $\begin{array}{c}54 \mathrm{M} \\
45 \mathrm{~V}\end{array}$ & 12 & 9 \\
$25-36 \mathrm{~m}$ & 73 & $\begin{array}{c}25 \mathrm{M} \\
48 \mathrm{~V}\end{array}$ & 9 & 6 \\
$37-48 \mathrm{~m}$ & 75 & $\begin{array}{l}32 \mathrm{M} \\
43 \mathrm{~V}\end{array}$ & 0 & 0 \\
\hline
\end{tabular}

Tal y como podemos ver en la tabla 1 del total de $n=298$ destacaban por las bajas puntuaciones obtenidas en la EDPRA 24 niños de los cuales 19 llegaron meses después a un servicio de atención temprana (SAT) por posibles trastornos en el desarrollo. Es importante resaltar que todos los participantes habían pasado el filtro para participar en la baremación, es decir no se conocía inicialmente que pudiera existir sospecha de una alteración que afecte al desarrollo comunicativo, lingüístico o cognitivo de los niños. Además, como ya se ha dicho, habían sido considerados como criterios de exclusión la existencia de deficiencia auditiva moderada o grave, deficiencia visual severa, síndrome de filiación genética, grandes prematuros, trastorno del neurodesarrollo, trastornos del sistema nervioso central, hospitalización prolongada, institucionalización durante los primeros meses de vida.

Los 19 niños que llegaron a posteriori al SAT podemos clasificarlos en tres grandes grupos: Trastornos de lenguaje, Trastornos de la comunicación, trastornos del vínculo de apego y otros trastornos del neurodesarrollo.

Los tiempos, en cuanto a detección precoz, logrados a partir de la aplicación de la EDPRA han sido significativamente inferiores a los obtenidos a través de los cribados de pediatría y de escuelas infantiles habituales ya que tal y como se muestra en la tabla 2 , de haber usado la EDPRA como herramienta de detección, los menores habrían obtenido una atención temprana más inmediata y, con ello, más eficaz.

Tabla 2.

Edad de detección de los trastornos del desarrollo a través de la escala EDPRA.

\begin{tabular}{|c|c|c|}
\hline $\begin{array}{l}\text { Edad detección } \\
\text { EDPRA }\end{array}$ & $\begin{array}{c}\text { Edad } \\
\text { detección otras } \\
\text { herramientas }\end{array}$ & $\begin{array}{r}\text { Meses de } \\
\text { diferencia }\end{array}$ \\
\hline 6 & 20 & 14 \\
\hline 8 & 22 & 14 \\
\hline 10 & 20 & 10 \\
\hline 10 & 12 & 2 \\
\hline 15 & 20 & 5 \\
\hline 16 & 20 & 4 \\
\hline 18 & 20 & 2 \\
\hline 21 & 28 & 7 \\
\hline 21 & 38 & 17 \\
\hline 23 & 28 & 5 \\
\hline 24 & 28 & 4 \\
\hline 24 & 30 & 6 \\
\hline 24 & 29 & 5 \\
\hline 25 & 36 & 11 \\
\hline 26 & 30 & 4 \\
\hline 29 & 35 & 6 \\
\hline 32 & 46 & 14 \\
\hline 33 & 35 & 3 \\
\hline 34 & 37 & 3 \\
\hline
\end{tabular}

En todos los casos, fueron los hitos de desarrollo pragmático registrados, los detectores más tempranos en alertar sobre una alteración o posible alteración en el desarrollo de los menores, llegando en algunos casos a adelantarse incluso más de un año a otros indicadores como pudieron ser los motores o de lenguaje a nivel léxico, sintáctico y/o semántico.

\section{Conclusión}

Los hitos de desarrollo pragmático poseen gran valor predictivo en el desarrollo infantil, no sólo en lo que compete a aspectos puramente comunicativos sino que su 
logro representa un importante marcador de la existencia de un desarrollo infantil armónico. Sabemos que son muchas y de diversas etiologías las variables que interfieren en el desarrollo de los infantes, pero creemos que deben ser de especial interés las ancladas en la socialización y comunicación del niño ya que estas unen al menor con todo aquello que le rodea pudiendo actuar como conector entre las distintas áreas objeto de atención temprana.

Tener en cuenta los marcadores pragmáticos nos ofrece una herramienta de gran valor en la detección precoz de las alteraciones del desarrollo y la investigación en esta línea nos aporta funcionalidad no solo a las evaluaciones sino también a las consiguientes intervenciones.

\section{Referencias}

Artigas-Pallars, J. (2007). Atención precoz de los trastornos del neurodesarrollo. A favor de la intervención precoz de los trastornos del neurodesarrollo. Rev Neurol, 44(3), S31-S34.

Botana, I., Peralbo, M. (2015). Estudio sobre la adaptacion y validación de un instrumento de evaluación del desarrollo pragmático temprano. Revista de estudios e investigación en psicología y educación, 9, 39-42.

http://dx.doi.org/10.17979/reipe.2015.0.09.374

Botana, I.,Vilameá, M. (2014). Atención temprana: papel del logopeda: ¿es la comunicación el área de mayor demanda?. Actas del XXIX Congreso de ALEFA 2014 (pp.152-158).Murcia:AELFA.

http://www.um.es/aelfa2014/docs/libro.pdf 